Systematic Review

\title{
Efficacy of Topical Applications on Reducing Uremic Pruritus: A Systematic Review
}

\section{Gabriel Wanda Sinawang, Rohmatul Faizah, Mohamad Roni Alfaqih and Andrik Hermanto}

Faculty of Nursing, Universitas Airlangga, Surabaya, Indonesia

\begin{abstract}
Introduction: Uremic pruritus is an unpleasant sensation that has been recognized as a frequent complication in patients with chronic kidney disease. This study was undertaken to determine the effect of topical applications for reducing pruritus.
\end{abstract}

Methods: A systematic review was conducted by searching Science Direct, Scopus and Google Scholar to create an integrative 12 articles review focusing on a 10 year period from 2009 to 2018 . The language used was English and the studies focused on pruritus, itching, hydration, chronic kidney disease and where the patient did not undergo a kidney transplant. The articles in this systematic review were experimental studies (pre-experimental, true experimental and quasi-experimental).

Results: Most of the findings of the studies showed that a topical application for reducing uremic pruritus (clove oil, almond oil, cromolyn sodium 4\%, baby oil, sweet almond oil, vinegar and avenasativa, glycerol and paraffin, sericin cream and chia seed oil) can be used as an adjuvant moisturizing agents for pruritis.

Conclusion: Topical applications have a positive effect on itching and decreased pruritus. Topical applications are simple, safe, inexpensive and easily applied, so it can be used on many people.

\section{ARTICLE HISTORY}

Received: December 26, 2019

Accepted: December 31, 2019

\section{KEYWORDS}

topical applications; uremic pruritus

\section{CONTACT}

Gabriel Wanda Sinawang

$\triangle$ gabriel.wanda.sinawang-

2018@fkp.unair.ac.id

$\equiv$ Faculty of Nursing, Universitas

Airlangga, Surabaya, Indonesia

Cite this as: Sinawang, G. W., Faizah, R., Alfaqih, M. R., \& Hermanto, A. (2019Efficacy of Topical Applications on Reducing Uremic Pruritus: A Systematic Review. Jurnal Ners, 14(3si), 252-255. doi:http://dx.doi.org/10.20473/jn.v14i3(si).17125

\section{INTRODUCTION}

Pruritus is one of the most common skin disorders among patients with end-stage renal disease, which disturbs their mental and physical health status. For instance, it causes sleep disorders, Although pruritus is not life-threatening, it can affect the quality of life of the patients (Karadag et al., 2014). Uremic pruritus is an unpleasant sensation that has been recognized as a frequent complication in patients with chronic kidney disease. It is one of the most common disabling symptoms in patients with end-stage renal disease. (Aramwit et al., 2012) It has been found that $15 \%$ to $49 \%$ of patients with chronic kidney disease and more than $40 \%$ of patients undergoing hemodialysis suffer from chronic pruritus. Pruritus frequency increases significantly alongside the deterioration of the disease and the treatment period. It can cause disturbances in the day and night rhythm, depression, sleeping disorders, anxiety and skin complications and it can also diminish quality of life (Nakhaee et al.,
2015).Patients with HD are at a high risk of experiencing drug toxicity due to kidney failure in terms of excreting drug metabolites. Non-chemical medications such as herbal medicines with low toxicity may be advised for such cases (Mehri, Afrasiabifar and Hosseini, 2018). The topical application of a herbal substance can moisturize the skin and reduce pruritus and this treatment can minimize the effect of chemicals for the patient with renal disease.

In this study, the researchers wanted to see the efficacy of topical applications when it comes to reducing uremic pruritus.

\section{MATERIALS AND METHODS}

\section{Research design}

This study used a systematic review with a questionbased search: "What is the effect of topical applications on reducing pruritus?" 


\section{Search strategy}

The trials to be included to the review were identified through electronic database searching from the earliest available time through to 2017 using the following databases: ScienceDirect, Scopus and Google Scholar by using the keywords "pruritus" and "chronic kidney disease".

\section{Inclusion and exclusion criteria}

The inclusion criteria in this review were experimental study and non-experimental studies that had been published from June 2009 to 2018, where the participants were 10 years old or more, where the language used was English and that focused on pruritus and chronic kidney disease. The exclusion criteria were studies that did not involve patients with chronic kidney disease and where there had been a transplant.

\section{Article searching process}

This systematic review was conducted according to the PRISMA (Preferred Reporting Items for Systematic Reviews and Meta-Analyses) guidelines. The database search was performed from 2009 to 2019. The literature research used three databases: ScienceDirect, SCOPUS and Google Scholar. The following key words were used: pruritus, itching, hydration, kidney disease and the patient did not undergo a kidney transplant. The limitations used in the literature search were that they had to be published in English. This study reviewed 12 articles. The articles come from Iran, Taiwan, Prances, Korea, Cairo and Thailand.

\section{RESULTS}

The review results showed the efficacy of the topical applications related to reducing uremic pruritus as presented in Table 1. Based on Table 1, the researchers were able to determine the efficacy of topical treatments for reducing uremic pruritus. There were 2 dominant applications found through natural and pharmaceutical therapy.

\section{Natural therapy}

From the 12 articles that reviewed, 9 articles used natural or non-pharmacological therapy as a comparison intervention. The interventions used baby oil, clove oil, sericin, chia seed and sweet almond oil. The applications were effective at moisturizing the skin because the substance was liquid paraffin (baby oil), contained essential fatty acids (sweet almond, chia seed), had moisturizing properties with a proinflamatory effect (sericin) and they contained natural oils (clove oil).

\section{Pharmacological therapy}

From the 12 articles that were reviewed, 3 articles used the pharmacological therapy as a comparison intervention. The intervention used cromolyn sodium $4 \%$, avena sativa and glycerol-parrafin. This application contains a chemical material that can decrease pruritus. Cromolyn sodium acts as a mast cell stabilizer for patient dialysis, avena sativa has the effect of moisturizing, protective, soothing and being an anti-inflammatory and glycerol-paraffin results in hydration and it is an anti-irritant.

This study was used to evaluate the efficacy of topical therapy using at least one of several instruments. The measuring instruments were ISS (Itch Severity Scale), the 5-D itch scale (duration, degree, direction, disability and distribution), VAS (Visual Analogue Scale), a Corneometer (skin parameters for hydration), a Mexameter (irritation and pigmentation), KDQOL-SF (Kidney Disease Quality of Life Short Form), DermaLab and Skin surface Phwas measured by the Skin Ph Meter PH900. There was also the D-Squame technique (the total surface area of all squames; [SURFT] the parameter measures the extent and density of the scales, the mean optical density without threshold and the [MOD] parameter measures the thickness of the scales, generic scale Short Form-12 (SF-12) questionnaire, the Dermatology Life Quality Index (DLQI), PSQI (The Pittsburgh Sleep Quality Index), the SF-36 Quality of Life Scale (SF-36 QoL), ODS (Overall Dry Scale), the Questionnaire of Pruritic Score and ItchyQol.

\section{DISCUSSION}

\section{Effectiveness of baby oil for treating uremic pruritus}

The severity of the itching was much lower in the participants treated with baby oil than those who were untreated. This was found in the study of (Lin et al., 2012), where the severity of the itching did not significantly differ between the groups treated with chilled baby oil and those treated with un-chilled baby oil. Chilled or un-chilled baby oil is effective as a cooling and soothing agent and moisturizing lotion. Furthermore, in a study conducted by (Karadag et al., 2014), itching improved the patient's quality of life and it reduced the sleep problems of the HD patients. Baby oil, which contains moisturizing raw coconut oil and oil with minerals, reduces itching through its effects such as stopping the transmission of the nerve fibers and decreasing chemical stimulus and inflammation. In another study (Mokhtarabadi et al., 2017), baby oil had moisturizing properties and there are similarities between this compound and physiological lipids, endogenous cannabinoids and emollients containing high water content.

\section{Effectiveness of topical clove oil for treating uremic pruritus}

Chronic pruritus can be broadly categorized into four major groups: dermatologic causes, systemic causes, neuropathic causes and psychogenic cause. There are several modalities for the treatment of pruritus including topical therapy, systemic therapy, phototherapy and behavioral therapy (Ibrahim et al., 2017). Clove oil (eugenol) is a topical anesthetic in dentistry and it might possess the potential to replace benzocaine as a topical agent. This therapeutic option for chronic pruritus has excellent results and it is free from toxic side effects. 
Table 1. List the Efficacy of Topical Applications on reducing Uremic Pruritus

\begin{tabular}{|c|c|c|}
\hline No & Article Writer & Efficacy of Topical Applications on reducing Uremic Pruritus \\
\hline 1. & Lin T, Lai Y, Guo S, Liu C, Tsai J, Guo H & $\begin{array}{l}\text { Chilled or un-chilled baby oil is effective as a moisturizing lotion and } \\
\text { cooling soothing agents. }\end{array}$ \\
\hline 2. & Ibrahim IM, Elsaie ML, Mohey-eddin MH & Natural oil is free from toxic side effects. \\
\hline 3. & $\begin{array}{l}\text { Aramwit } \mathrm{P}, \text { Keongamaroon } \mathrm{O} \text {, } \\
\text { Siritientong T, Bang N }\end{array}$ & $\begin{array}{l}\text { Sericin cream can also significantly increase skin hydration and } \\
\text { reduce skin irritation and skin pigmentation in patients. }\end{array}$ \\
\hline 4. & $\begin{array}{l}\text { Jeong SK, Ph D, Park HJ, Ph D, Park BD, } \\
\text { Ph D, et al }\end{array}$ & $\begin{array}{l}\text { Chia seed oil is effective at treating pruritus and xerosis and it is also } \\
\text { beneficial for skin moisturizing. }\end{array}$ \\
\hline 5. & $\begin{array}{l}\text { Balaskas E, Szepietowski JC, Bessis D, } \\
\text { Ioannides D, Ponticelli C, Ghienne C. } \\
\text { Randomized }\end{array}$ & $\begin{array}{l}\text { The effective emollient treatment of uremic xerosis can also } \\
\text { efficiently relieve uremic pruritus. }\end{array}$ \\
\hline 6. & Nakhaee S, Nasiri A, Waghei Y & Vinegar and Avena sativa lotion can be used for uremic pruritus. \\
\hline 7. & Karadag E, Kilic SP, Karatay G, Metin O & $\begin{array}{l}\text { Baby oil had short term positive effects on itching, sleep quality and } \\
\text { quality of life in the HD patients who had itching complaints. }\end{array}$ \\
\hline 8. & Tricaesario C, Widayati RI, Oil A & $\begin{array}{l}4 \% \text { almond oil cream is effective at increasing the skin's moisture } \\
\text { level. }\end{array}$ \\
\hline 9. & $\begin{array}{l}\text { Maryam K, Feily A, Dormanesh B, } \\
\text { Ghorbani A }\end{array}$ & $\begin{array}{l}\text { CS } 4 \% \text { cream was more effective at reducing pruritus than the } \\
\text { placebo. }\end{array}$ \\
\hline 10. & Mehri Z, Afrasiabifar A, Hosseini N & $\begin{array}{l}\text { Topical application of sweet almond oil improved itchiness in patient } \\
\text { with uremic pruritus. }\end{array}$ \\
\hline 11. & $\begin{array}{l}\text { Mokhtarabadi S, Shahabinejad M, } \\
\text { Sadeghi T, Kazemi M }\end{array}$ & $\begin{array}{l}\text { Thermal changes in baby oil had no effect on assuaging pruritus } \\
\text { severity, and the only effective factor was its moisturizing properties. }\end{array}$ \\
\hline 12. & (ArdashirAfrasiabifar, et al & $\begin{array}{l}\text { Sweet almond oil as a natural method without complications could } \\
\text { be a non-invasive way to help patients to reduce uremic pruritus. }\end{array}$ \\
\hline
\end{tabular}

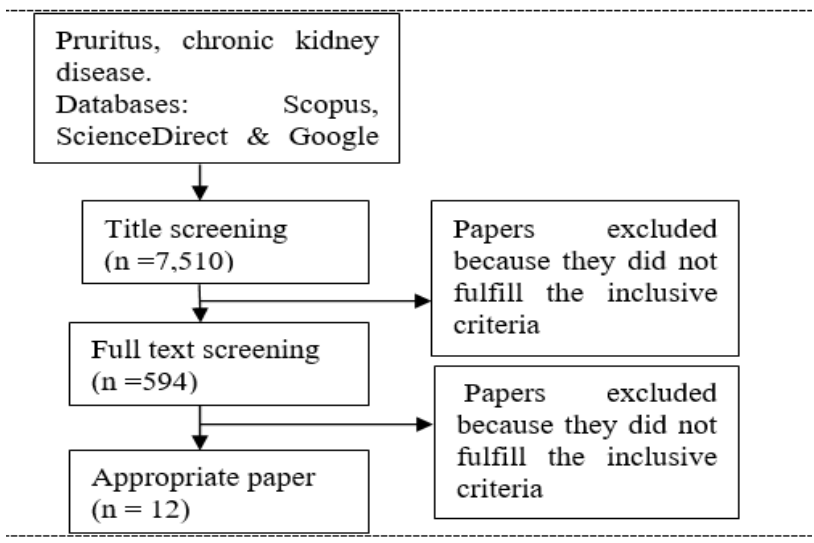

Figure 1. Flowchart of the research on pruritus and chronic kidney disease

\section{Effectiveness of topical Avena Sativa, Vinegar and Hydroxyzine for treating uremic pruritus}

The study of (Nakhaee et al., 2015) showed that Avena sativa produced a protective moisturizing barrier on the skin which helped to soften and moisten the skin and heal tissue, hence reducing pruritus. Vinegar has been reported to be useful for the treatment of pruritus. By maintaining the acidic $\mathrm{pH}$ of the skin's surface, vinegar helps to preserve the skin's barrier function and thus it reduces skin irritation. Low pH topical therapies decrease pruritus by limiting the activity of serine proteases on the skin nerve fibers. On the other hand, pruritus is cause by the elimination of urea and sodium through the skin. A diluted vinegar solution can counteract the urea crystals and reduce pruritus.

\section{Effectiveness of Sericin Cream for treating uremic pruritus}

The level of skin hydration in the patients' extremities increased after treatment with sericin[3]. Sericin cream and the cream base were used to prepare the sericin cream that increased moisture content of the stratum corneum. However, the level of hydration was significantly higher in the skin treated with sericin cream.

\section{Effectiveness of topical chia seed oil for treating uremic pruritus}

Skin capacitance, which represents the skin's hydration, was below the normal values before the treatment and it gradually increased during the treatment time with the chia seed oil containing moisturizer (Jeong et al., 2010).

\section{Effectiveness of topical cromolyn sodium $4 \%$ for treating uremic pruritus}

This study found out that in the third and fourth week of the study, CS 4\% cream was more effective at reducing pruritus than the placebo (Maryam et al., 2013). The skin of the CKD patients with pruritus had a greater number of mast cells and also an increased plasma histamine level compared to those without 
pruritus, so it is logical to use the mast cell stabilizing $\mathrm{CS}$ in the treatment of renal pruritus.

\section{Effectiveness of topical glycerol and paraffin for treating uremic pruritus}

The results indicate that an effective emollient treatment of uremic xerosis can also efficiently relieve uremic pruritus (Balaskas et al., 2011). The effects of an emollient and skin protective product combining glycerol $15 \%$ and paraffin $10 \%$ were rapid hydrating and a smoothing effect.

\section{Effectiveness of sweet almond oil for treating uremic pruritus}

One of the natural agents believed to have a moisturizing effect is almond oil due to its occlusive and emollient properties ('EFEKTIVITAS KRIM ALMOND OIL 4\% TERHADAP TINGKAT KELEMBAPAN KULIT', 2016). Furthermore, in a study conducted by (Mehri, Afrasiabifar and Hosseini, 2018), dry skin (xerosis) is introduced as a cause of uremic pruritus. Topical agents such as sweet almond oil containing essential fatty acids such as linoleic acid may help in its relief through increasing the leukotrienes and prostaglandins, which moisturize the skin. In addition, essential fatty acids, by reducing the lymphokines and lymphocytes, may lead to the reduction of pruritus and dermatological inflammations. For instance, arachidonic and linoleic acids play a protective role in the skin and their deficiency in patients undergoing HD may lead to dermatological complaints such as pruritus. (Afrasiabifar, Mehri and Hosseini, 2017) In justifying the effectiveness of sweet almond oil on the relief of uremic pruritus, it can be said that sweet almond contains essential fatty acids such as linoleic acid that have been shown to be effective in relieving pruritus.

\section{The Implication toward Practice}

By recognizing several of the topical treatments that can be given to patients with kidney failure who have uremic pruritus, nurses are expected to be able to carry out the role of nursing in collaboration with doctors and pharmacists in providing natural or chemical treatment options.

\section{CONCLUSION}

Pruritus is an effect of kidney disease which will affect the quality of life of the sufferer. Topical therapy is one of the options for reducing the impact of pruritus. Natural topical therapies and chemicals generally aim to hydrate by providing moisture to the skin of the patients with renal failure, who tend to dry out. Some topical therapies have additional functions such as having an anti-irritating and anti-inflammatory effect. Topical therapy is an invasive action and it is a simple therapy that can be applied independently. Topical therapy (especially natural therapy) can be an option because it is effective, efficient and easily accessible by all groups.

\section{REFERENCES}

Afrasiabifar, A., Mehri, Z. and Hosseini, N. (2017) 'Efficacy of topical application of sweet almond oil on reducing uremic pruritus in hemodialysis patients: A randomized clinical trial study', Iranian Red Crescent Medical Journal. doi: 10.5812/ircmj.34695.

Aramwit, P. et al. (2012) 'Sericin cream reduces pruritus in hemodialysis patients: a randomized, double-blind, placebo-controlled experimental study.', BMC nephrology. doi: 10.1186/14712369-13-119.

Balaskas, E. et al. (2011) 'Randomized, double-blind study with glycerol and paraffin in uremic xerosis', Clinical Journal of the American Society of Nephrology. doi: 10.2215/CJN.05490610.

'EFEKTIVITAS KRIM ALMOND OIL 4\% TERHADAP TINGKAT KELEMBAPAN KULIT' (2016) JURNAL KEDOKTERAN DIPONEGORO.

Ibrahim, I. M. et al. (2017) 'Effectiveness of topical clove oil on symptomatic treatment of chronic pruritus', Journal of Cosmetic Dermatology. doi: 10.1111/jocd.12342.

Jeong, S. K. et al. (2010) 'Effectiveness of topical chia seed oil on pruritus of end-stage renal disease (ESRD) patients and healthy volunteers', Annals of Dermatology. doi: 10.5021/ad.2010.22.2.143.

Karadag, E. et al. (2014) 'Effect of baby oil on pruritus, sleep quality, and quality of life in hemodialysis patients: Pretest-post-test model with control groups', Japan Journal of Nursing Science. doi: 10.1111/jjns.12019.

Lin, T. C. et al. (2012) 'Baby oil therapy for uremic pruritus in haemodialysis patients', Journal of Clinical Nursing. doi: 10.1111/j.13652702.2011.03906.x.

Maryam, K. et al. (2013) 'Efficacy of topical cromolyn sodium $4 \%$ on pruritus of uremic nephrogenic patients ; a randomized double blind study on 60 patients', (March). doi: 10.5414/CP201629.

Mehri, Z., Afrasiabifar, A. and Hosseini, N. (2018) 'Improved Itchy Quality of Life Following Topical Application of Sweet Almond Oil in Patients with Uremic Pruritus: A Randomized, Controlled Trial', Jundishapur Journal of Chronic Disease Care. doi: $10.5812 /$ jjcdc.68164.

Mokhtarabadi, S. et al. (2017) 'The Effect of Administration of Baby Oil on the Severity of Pruritus in Hemodialysis Patients', MedicalSurgical Nursing Journal. doi: 10.15171/jrip.2017.22.

Nakhaee, S. et al. (2015) 'Comparison of Avena Sativa, vinegar, and hydroxyzine for uremic pruritus of hemodialysis patients a crossover randomized clinical trial', Iranian Journal of Kidney Diseases. 